\title{
Editorial
}

\section{Complex Systems in Aesthetics and Arts}

\author{
Juan Romero $\left(\mathbb{D},{ }^{1}\right.$ Colin Johnson $(\mathbb{D}),{ }^{2}$ and Jon McCormack $(\mathbb{D})^{3}$ \\ ${ }^{1}$ University of A Coruña, A Coruña, Spain \\ ${ }^{2}$ University of Kent, Canterbury, UK \\ ${ }^{3}$ Monash University, Melbourne, Australia \\ Correspondence should be addressed to Juan Romero; jj@udc.es
}

Received 16 April 2019; Accepted 16 April 2019; Published 2 May 2019

Copyright (c) 2019 Juan Romero et al. This is an open access article distributed under the Creative Commons Attribution License, which permits unrestricted use, distribution, and reproduction in any medium, provided the original work is properly cited.

\begin{abstract}
The arts are one of the most complex of human endeavours, and so it is fitting that a special issue on Complex Systems in Aesthetics and Arts is being published. As the editors of this special issue, we would like to thank the reviewers of the submitted papers for their hard work in making this issue possible, as well as the authors who submitted their work and were very responsive to the comments of the reviewers and editors.
\end{abstract}

The word complexity has a specific meaning in the context of "complex systems" research, as the study of systems made of many components-not in themselves necessarily complex - that through loosely coupled, local interactions generate complex, emergent behaviours. Such systems have the potential to act as the basis for the production of artworks, whether entirely computer generated or as a result of a cocreative system between humans and computers. Such art might make its impact through the intrinsic interest of the complex behaviour in the system, by representing, exploring, or connoting some worldly aspect of complexity, or by using complex systems as a way of exploring a space of possible works. Furthermore, complex systems research has the potential to simulate emergent processes in the artworld, such as the interaction between artists, audiences, and critics, or the development of aesthetic ideas or artistic fashions over time.

The context for the special issue is explored in the first paper, Understanding Aesthetics and Fitness Measures in Evolutionary Art Systems, authored as an overview paper on the topic by the editors and I. Santos. This takes a particular algorithm that is grounded in complexity science ideas-evolutionary search-and explores links between the construction of fitness measures in these systems, and measures and concepts of aesthetic value from the philosophy and psychology of art. A common feature of complex systems is that individual agents make evaluations as a driver for behaviour, and so the links formed in this paper between the human behaviour of making aesthetic judgements and similar processes in computer systems have the potential to inform work in many applications of complex systems to the arts.

This theme of aesthetic measures is continued in the paper by A. Carballal et al., Avoiding the Inherent Limitations in Datasets Used for Measuring Aesthetics When Using a Machine Learning Approach. In this paper, the authors explore how well a machine learning approach can replicate the aesthetic and quality judgements of a number of humans across a large set of photographs, exploring whether the machine learning algorithms can learn to replicate and generalise from human judgements and then apply these accurately to new examples. The paper also addresses whether the learned models replicate the phenomenon found in the human results whereby aesthetic value and technical quality are correlated. They conclude that the correlation is present also in the learned models, though less strongly than with humans, and that the machine learning models were typically better at assessing (more objective) technical quality than (more subjective) aesthetic value.

The remaining articles explore a variety of other topics concerned with aesthetic aspects of images and graphics. Evolutionary Computation for Modelling Social Traits in Realistic Looking Synthetic Faces by F. Fuentes-Hurtado and colleagues explores the use of evolutionary computation to select sets 
of facial features that convey a particular social emotion and then uses an automated image editing approach, Poisson Image Editing, to create a realistic composite image that combines the chosen features. By contrast with the realistic images in that paper, Image Evolution Using $2 D$ Power Spectra by M. Gircys and B. J. Ross uses evolutionary algorithms to produce abstract artworks based on realistic photographs and paintings. The system is based on a spectral analysis of the original image, which is used to construct a fitness function that then drives the evolutionary process to generate novel images based on the same spectral profile. The system produces images that still connote features of the source image but are more abstract.

Finally, the paper Evolving Stencils for Typefaces: Combining Machine Learning, User's Preferences and Novelty, by T. Martins et al., explores a system with two components. The first of these is an evolutionary system for exploring the complex search space of typefaces. The second component is a human-computer cocreative system to develop the fitness function that is used by the evolutionary algorithm. The paper demonstrates an exemplary piece of work in combining human and computer expertise in a complex aesthetic domain.

We believe that this selection of articles offers an interesting and timely insight into the interactions between aesthetics, machine learning, and computational creativity. We hope that you enjoy and learn from reading the papers in this issue.

\section{Conflicts of Interest}

The editors have no conflict of interest regarding the publication of this special issue.

Juan Romero

Colin Johnson

Jon McCormack 


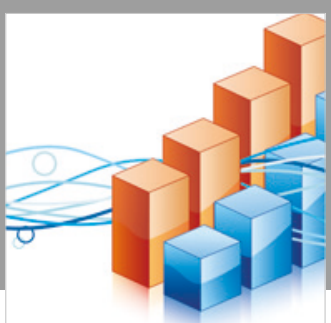

Advances in

Operations Research

\section{-n-m}
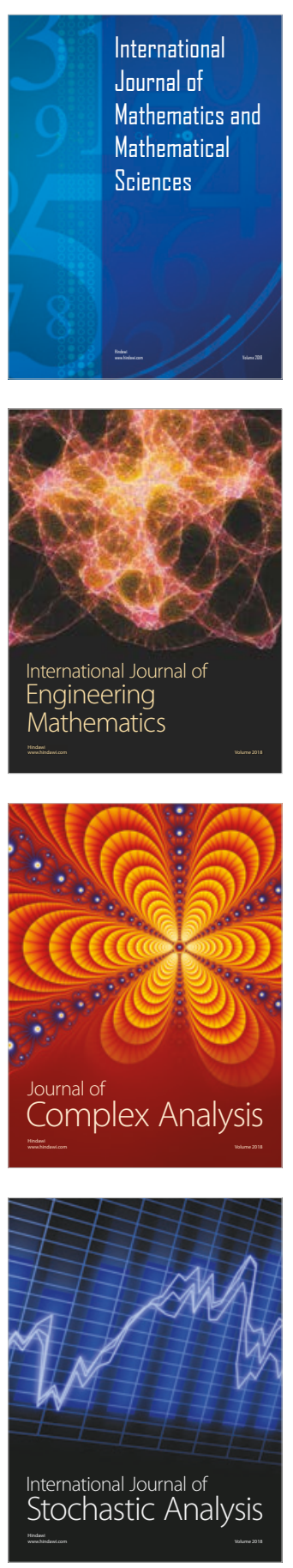
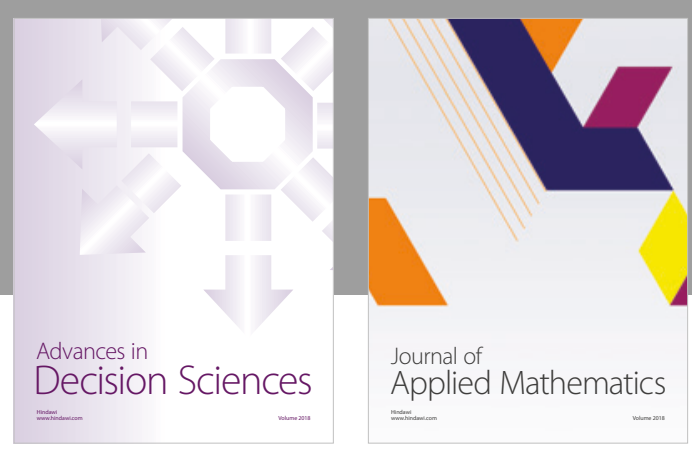

Journal of

Applied Mathematics


Submit your manuscripts at

www.hindawi.com

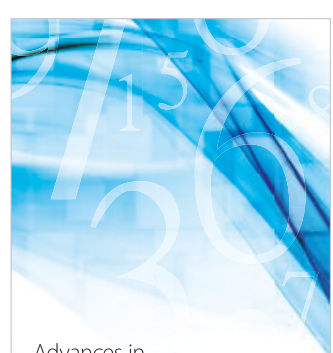

Advances in
Numerical Analysis
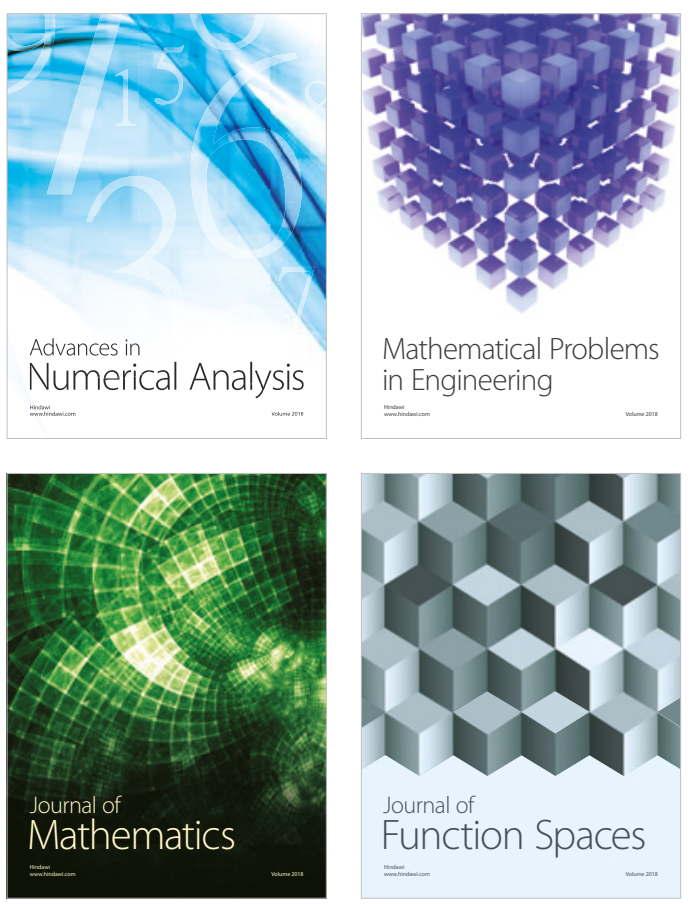

Mathematical Problems in Engineering

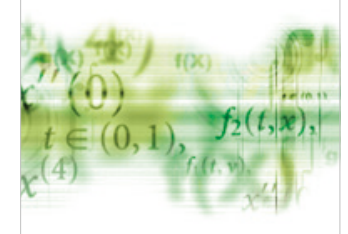

International Journal of

Differential Equations

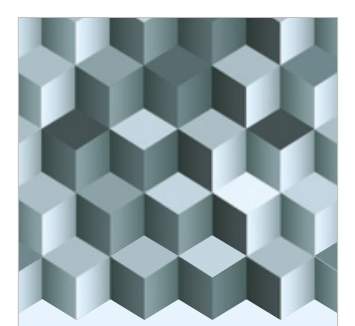

Journal of

Function Spaces
The Scientific

World Journal

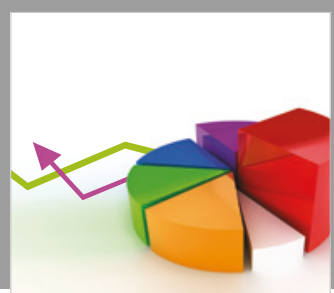

Journal of

Probability and Statistics
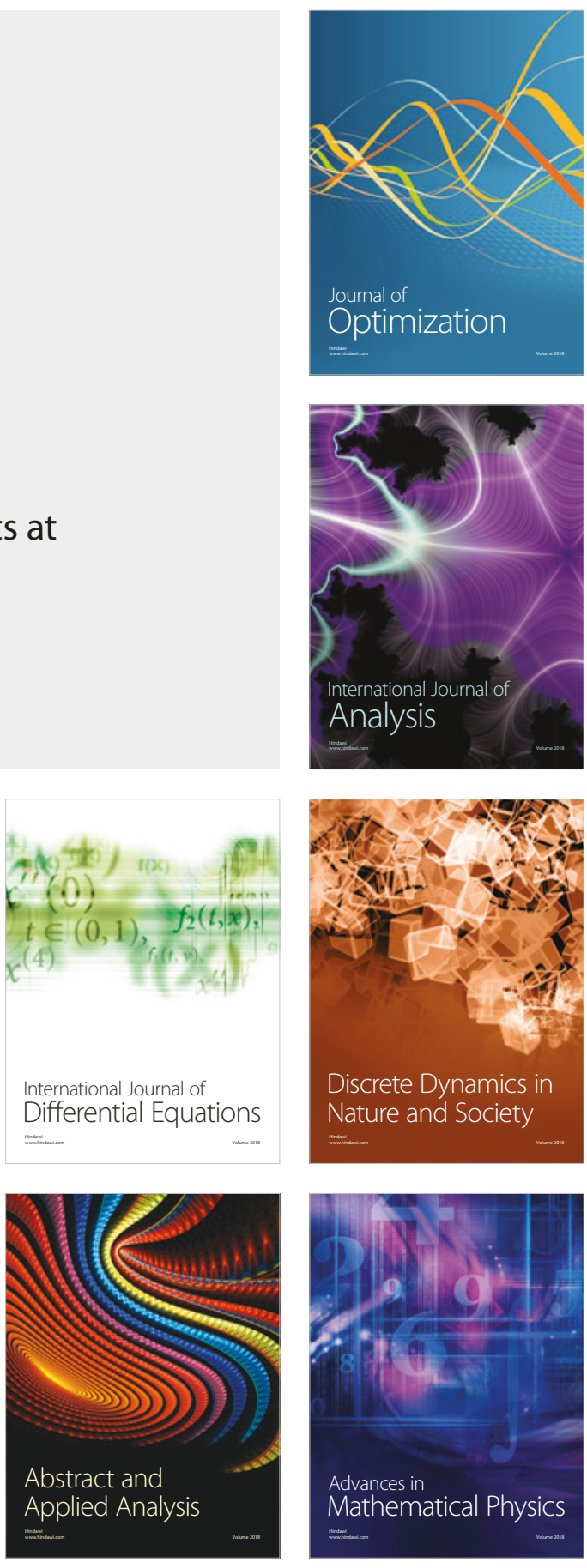